exhibited a perfectly closed sagittal suture, and which was not far removed from this variety of skull-form. Of these thirty-one skulls, six showed a parietal tuber nearer the squamal than the sagittal margin; and two had this remnant of the ossific centre palpably nearer to the lambdoidal than to the coronal edge.

From these observations, we may conclude that there is no typical or fixed point in Nature to which the development of the ossific centre is confined; and secondly, that the nearer to the mesial line the original centre may be, the more rapidly will the bones on each side approach to the sagittal suture; and, if the two centres had been originally placed within an unusually short distance of each other, the result would naturally be the early contact of the inner margins, which would predispose to synostosis on this line. Growth being checked along this line, an increased stimulus is given to ossification in all other directions, so the posterior extension of the parietal progresses to compensate for the checked growth transversely. Meeting with the ascending squama occipitis, however, causes another alteration of action; and the result of the bone-growth along the edges of these opposing elements is the prominent occipital angle projecting backwards. Forwards, the area of ossification proceeds undisturbed until it meets the frontal; and, as the inward and backward checks have caused an increased vital rapidity of bone growth in this mesial bone, the biparietal extends so rapidly that its confluent angles stretch into the anterior fon ianelle. Admitting this theory, that the scaphocephalic form of skull arises from the presence of two ossific centres placed unusually near to the mesial line, so close that synostosis takes place at an early period, causing a coalescence imilar to that in the twin centres of the squama occipitis, we have an easy explanation of all the points doubtful or discrepant in the various descriptions; we can understand how traces of tubera may exist even in true scaphocephali; and also how, in the example of Daniel B., published by Minchin, the radii seem to spring from a common centre; that centre is evidently broader transversely than antero-posteriorly, and is formed of two lateral points centrally coalescing. It also helps us to understand the existence of the carina inexplicable otherwise on the single centre theory.

\section{ON AFFECTIONS OF VASO-MOTOR NERVES.} BY JOHN W. OGLE, M.D., Physician to St. George's Hospital.

At page 264 of this Journal for Aug. 22nd last, I notice an abstract of Dr. J. Russell Reynolds' paper, at the recent Oxford meeting of the British Medical Association, on Certain Affections of the Vaso-motor System of Nerves. Dr. Reynolds related an instance in which the right radial pulse could be arrested by pressure on the lower cervical vertebra, at the same time pain in the back and an uneasy feeling in the right arm being occasioned; and with these phenomena he compared certain cases of paroxysmal sensations in the limbs, with which were associated irregularity, intermission, or weakness of the pulse of the affected side. "Dr. Reynolds was of opinion that such symptoms resembled epileptic seizures."

Not having had the advantage of being present when the paper was read, I had no opportunity of quoting the following case; which may well be adduced, I think, in illustration of the subject of the paper in question, and is in itself so remarkable and interesting that it ought to be kept in mind by all persons studying affections of the nervous system.

The case is related by Dr. Pereira, in his work upon the Materia Medica; and was under the care of Dr. Holst, Professor in the University of Christiania. It was that of an epileptic, in which it was observed that the pulse in one arm was always imperceptible during the epileptic paroxysm. "On a post mortem examination, it was discovered that an anomalous distribution of the arteries existed, so that this arm was supplied with blood by the vertebral arteries, which derived it (the blood), through the basilar artery, from the carotids. The cessation of the pulse during the paroxysm proved that the circulation through these vesseds was temporarily interrupted."

Your readers will be aware that observation and experiment led Dr. Brown-Séquard to conclude that certain of the phenomena of epilepsy depend upon reflex contraction in the blood-vessels, and especially the small arteries of the brain, owing to irritation of the origin of the sympathetic nerve animating them, arising from certain direct or indirect nutritional changes in the cerebro-spinal axis. My friend, Dr. E. Fox has also (in vol. ii of the St. George's Hospital Reports) strongly advocated arterial spasm as the proximate cause of most of the phenomena of epilepsy.

Is not the exceptional case which I have above cited-observed, as it was, many years before Dr. Brown-Séquard's suggestion-in harmony with this view, and, indeed, to a certain extent, corroborative of it, as also of the suggestion made by Dr. Reynolds?

In conclusion, I would draw attention to a case recorded by myself in the twelfth volume of the Pathological Society's Transuctions, pp. 2-4. The case was one of apoplexy, in connection with slight softening of, and extravasation of blood within, the pons Varolii, and clots in the right corpus striatum and right cerebral hemisphere. Among other symptoms, peculiar tetanic spasms of all the limbs existed, intensified during venesection; and also violent pulsations of the right carotid artery and right temporal arteries, the action of the left carotid artery being scarcely felt. Moreover, the condition of the pupils was remarkable at one time, the right one being dilated and oval, and later on the left one being the most dilated and also oval. The eyeballs were remarkable, as being very prominent; the prominence increasing before death so as to separate the eyelids. I suggested the question at the time, how far the difference in the pulsation of the vessels in the neck was owing to modifications of vaso-motor influence; and the same might, I think, be asked of the alterations in the pupil and of the prominence of the eyeballs.

\section{ON ERGOT OF RYE IN THE TREATMENT OF NEURALGIA.*}

By EDWARD WOAKES, M.D.Lond., F.L.S., Luton.

IN the October number of the Fournal of Cutaneous Medicine for 1867 , I published a paper on the Correlation of Neuralgia to Skin-Rash, believing that the consideration of some of the typical forms of this association of symptoms, in which the phenomena are to a certain extent exposed to accurate observation, wonld tend to throw light on the more obscure, though perhaps more frequently occurring, forms of neuralgia. One inference arrived at was, that the rash, when present in neurotic disease, can be accounted for only by a reference to the structural anatomy of the parts involved in the lesion; while the uncertain fact of its occurrence was inferred to depend upon the nature and intensity of the exciting cause of the attack. In other words, the rash was looked upon as complementary of the pain, but having this important distinction, that, being a tangible symptom, whose causation could be traced, it suggested a clue whereby to unravel its more obscure and more formidable congener, the pain.

In order to make these remarks intelligible, I will, with your permission, briefly recapitulate so much of the theory as is necessary to show the connexion between it and the treatment now proposed, which results as a direct corollary from it.

A comparison of the neuralgic and eruptive phenomena which occur when a nerve is wounded, with the corresponding symptoms exhibited in purely idiopathic cases of nerve-lesion, showed so close an approximation as to permit of their being regarded, for all practical purposes, as identical. The theory developed from this parallelism of symptoms. was this:-The results being similar in both the traumatic and idiopathic classes, the causation in each group of cases, though differing in respect of intensity, operates through the same anatomical channels, and induces the same physiological phenomena in both.

In the traumatic instances, the symptoms were shown to be manifestly due to the paralysing influence of the injury or shock upon the fibres of the sympathetic system of nerves accompanying the arteries in their distribution to the skin and to the sentient nerves; and the resulting symptoms were found to be in accordance with the physiological sequence of such palsy.

In the other instance, that of idiopathic neuralgia associated with skin-rash, of which shingles may be taken as the type, the same theory of shock inducing palsy of the sympathetic fibres supplying the affected tissues was cited to explain the attendant phenomena. The sympathetic nerve-fibres accompanying, say, an intercostal artery, are lost in the direction of the skin, on the minute divisions of the artery supplying its ultimate structure. When these nerve-fibres are paralysed from any cause, their function of regulating the calibre of the vessels will be withdrawn. Passive congestion of these vessels, and consequent effusion of liquor sanguinis, are the physiological consequences of this nerve-palsy. Tracing such an effusion in a cuticular papilla, there will be found, in the first place, a corresponding elevation of the cuticle covering it, which gradually gains the character of a true vesicle as the effusion increases, until in this manner a spot of herpes is produced. Following out the track of this anatomical clue, we are led to an explanation of the pain - the neuralgia - so often associated with shingles. The same artery which supplies the affected spot of skin, sends also nutrient

* Read in the Medical Section at the Annual Meeting of the British Medical Association in Oxford, August 1868. 